\title{
THRESHOLDS FOR MACROPARASITE INFECTIONS
}

\author{
ANDREA PUGLIESE AND LORENZA TONETTO
}

\begin{abstract}
We analyse here the equilibria of an infinite system of partial differential equations modelling the dynamics of a population infected by macroparasites. We find that it is possible to define a reproduction number $R_{0}$ that satisfies the intuitive definition, and yields a sharp threshold in the behaviour of the system: when $R_{0}<1$, the parasite-free equilibrium (PFE) is asymptotically stable and there are no endemic equilibria; when $R_{0}>1$, the PFE is unstable and there exists a unique endemic equilibrium. The results mainly confirm what had been obtained in simplified models, except for the fact that no backwards bifurcation occur in this model. The stability of equilibria is established by extending an abstract linearization principle and by analysing the spectra of appropriate operators.
\end{abstract}

\section{INTRODUCTION}

The fundamental role of parasites in structuring animal communities is now recognized by most scientists and textbooks [13]. Mathematical models have helped in the understanding of the interactions among hosts and parasites, especially in finding the conditions for host regulation, and for parasite establishment. In models for the so-called micro-parasites (bacteria, viruses,... ), the latter is often expressed in terms of the reproductive number $R_{0}$, the expected number of infected hosts produced by a single infected host in a completely susceptible host population [8]: in fact, in most epidemic models, $R_{0}>1$ is a necessary and sufficient condition for the instability of the disease-free equilibrium, and a sufficient condition for the persistence of pathogens.

A similar concept (see, for instance, [24]) has been introduced in several models for the so-called macro-parasites (mainly helminths). However, the basic models for macro-parasites consist of an infinite system of differential equations for which stability conditions of parasite-free equilibria have not been rigorously established so far. On the other hand, the stability conditions have been obtained in lowdimensional approximate models $[1,22]$ which may give somewhat different results according to the approximation [19, 25]; the analysis of an infinite-dimensional model has been however performed by Kretzschmar [18] as further discussed below.

The starting point for modelling macroparasites is the model first presented by Kostizin [16], in which the main variables are $p_{i}(t)$, the density of hosts carrying $i$ parasites.

Parasites in one host may increase from $i-1$ to $i$ because of new infections at rate $\varphi(t)$; may decrease from $i+1$ to $i$ because of the death of one parasite: it will 
be assumed that each parasite dies (independently of the number of parasites in the same host) at rate $\sigma$.

Hosts may also be born and die: it will always be assumed that newborn hosts are parasite-free. It may also be assumed that hosts' fertility is reduced by parasites: the simplest consistent law is a multiplicative law [9], so that fertility of a host carrying $i$ parasites is reduced by a factor $\xi^{i}$ with $0<\xi \leq 1$. As for host mortality, the simplest assumption is that hosts are subject to natural mortality plus an additional rate $\alpha$ for each parasite harboured.

Under these assumptions, one obtains the following infinite system of differential equations

$$
\left\{\begin{array}{l}
\frac{d}{d t} p_{0}(t)=-(\mu+\varphi(t)) p_{0}(t)+\sigma p_{1}(t)+b \sum_{i=0}^{+\infty} p_{i}(t) \xi^{i} \\
\frac{d}{d t} p_{i}(t)=-(\mu+\varphi+i(\alpha+\sigma)) p_{i}(t)+\sigma(i+1) p_{i+1}(t)+\varphi p_{i-1}(t) \quad i \geq 1 .
\end{array}\right.
$$

where it has been assumed that natural birth rate $b$ and death rate $\mu$ are independent of host density.

Kreztschmar [18] has analysed this system under the assumption

$$
\varphi(t)=\frac{h P(t)}{c+N(t)}
$$

where $N(t)=\sum_{i=0}^{\infty} p_{i}(t)$ is the total density of hosts and $P(t)=\sum_{i=0}^{\infty} i p_{i}(t)$ is the total density of adult parasites. This form of $\varphi$ can be obtained from a sub-model that includes infection through free-living larvae [1].

She studied the system for $c=0$ (discussed in Section 6) and $c>0$. In the latter case, she found a sufficient condition (necessary and sufficient when $\xi=1$ ) for the existence of equilibria with parasites. It is not easy to interpret biologically this condition. Moreover, it cannot be written as a reproduction number at the parasite-free equilibrium, since no parasite-free equilibria exist; in fact, in that model, the host population, in absence of parasites, grows (or decreases) exponentially.

In order to have parasite-free equilibria, it is necessary to introduce densitydependence in hosts' fertility and/or mortality. For the sake of simplicity, here we restrict ourselves to assume density-dependence in fertility, and density-independent mortality. When a generic function for density-dependence is used, the method of generating function, first used by Hadeler and Dietz [12] and then by Kretzschmar $[17,18]$, seems not to be helpful. We therefore chose to use methods based on perturbation of linear semigroups. Moreover, we found that including hosts' age in the model (as in [17]) does not really introduce big complications, and indeed makes many expressions more transparent. 
Hence, we allow for age-dependent host fertility and mortality, and arrive at the following system of differential equations:

$$
\left\{\begin{aligned}
\frac{\partial}{\partial t} p_{i}(a, t)+ & \frac{\partial}{\partial a} p_{i}(a, t)=-(\mu(a)+\varphi(t)+i(\alpha+\sigma)) p_{i}(a, t) \\
& +\sigma(i+1) p_{i+1}(a, t)+\varphi(t) p_{i-1}(a, t) \quad i \geq 0 \\
p_{0}(0, t)= & \psi(N(t)) \int_{0}^{+\infty} \beta(a) \sum_{i=0}^{+\infty} p_{i}(a, t) \xi^{i} d a \\
p_{i}(0, t)= & 0 \quad i>0 \\
p_{i}(a, 0) & =h_{i}(a) \quad i \geq 0
\end{aligned}\right.
$$

with the convention $p_{-1}(a, t) \equiv 0$. Here $p_{i}(a, t)$ for $i \geq 0$ and $a$ in $[0,+\infty)$ denotes the density of hosts of age $a$ harbouring $i$ parasites at time $t$.

Here again the infection rate $\varphi(t)$ is given by (1.2), with

$$
N(t)=\int_{0}^{+\infty} \sum_{i=0}^{+\infty} p_{i}(a, t) d a \quad \text { and } \quad P(t)=\int_{0}^{+\infty} \sum_{i=1}^{+\infty} i p_{i}(a, t) d a .
$$

As for demographic parameters, $\mu(a)$ is the natural death rate of hosts while $\alpha$ is the additional death rate for each parasite carried. The fertility rate of hosts carrying $i$ parasites is given by $\psi(N(t)) \beta(a) \xi^{i}$, where $\psi$ is a decreasing function that shapes the density dependence of fertility.

Under standard conditions (see, for instance, [14]), this system will have a parasite-free equilibrium (PFE) at the hosts' carrying capacity. The reproduction number $R_{0}$ can be defined as the expected number, when hosts are at the $\mathrm{PFE}$, of successful infecting larvae produced in its life by a newly established adult parasite. In this paper, we show that this quantity defines a threshold for this model: if $R_{0}>1$, there exists an equilibrium with a positive number of parasites, and the PFE is unstable; if $R_{0}<1$, the parasite-free equilibrium is asymptotically stable for (1.3) (if it is so for the purely demographic equation), and there are no positive equilibria.

The organization of the paper is as follows.

In Section 2 we state the exact assumptions on the vital rates. In Section 3 we study the existence of positive equilibria, and show that this is equivalent to $R_{0}>1$. In Section 4, we set this model in an abstract framework, stating the well-posedness result obtained in [23]; furthermore, we prove, with the help of a theorem due to Desch and Schappacher [5], a general linearization principle for equilibrium stability in this class of equations. In Section 5 we go back to (1.3), showing that the growth rate $\omega$ of the linearized, at the PFE, semigroup is negative (hence the PFE is exponentially asymptotically stable) if $R_{0}<1$; and is positive (hence the PFE is unstable) if $R_{0}>1$. Finally, in the last section we discuss the biological interpretation of the results, their connection with the literature, and some possible extensions. 


\section{Preliminary ASSUmptions}

In order to perform a qualitative study of system (1.3) (as well as in [23] to prove existence and uniqueness of solutions), we take the following assumptions (see for instance [27]):

(H1) $\mu$ is a nonnegative, locally integrable function and there exist values $\mu_{-}, \mu_{+}$ such that $0<\mu_{-} \leq \mu(a) \leq \mu_{+}$for a.e. $a \in[0,+\infty)$

(H2) $\beta \in L^{\infty}[0,+\infty), \beta(a) \geq 0$.

Concerning the function $\psi$ decribing density-dependence in host fertility, we assume

(H3) $\psi \in C^{1}([0,+\infty)), \psi(0)=1, \psi^{\prime}(s)<0, \lim _{s \rightarrow+\infty} \psi(s)=0$.

Note that $\psi(0)=1$ is simply a normalization, since any constant can be inserted in the function $\beta$.

Another condition is needed to obtain a parasite-free stationary solution of (1.3). If $p=\left(p_{0}(a), p_{1}(a), \ldots\right)$ is a stationary solution of $(1.3)$ corresponding to $\varphi=0$, then $p_{i}(a) \equiv 0$ for $i>0$ and $p_{0}(a)=p_{0}(0) \pi(a)$ where

$$
\pi(a)=e^{-\int_{0}^{a} \mu(s) d s} .
$$

Setting

$$
\mathcal{R}=\int_{0}^{+\infty} \beta(a) \pi(a) d a,
$$

it can be easily seen that there is a stationary solution with $\varphi=0$ if and only if there exists $K>0$ such that

$$
\psi(K)=\frac{1}{\mathcal{R}}
$$

that is, because of (H3), if and only if

(H4) $\mathcal{R}>1$

If $\mathcal{R} \leq 1$, it is not difficult to show that the host population will decrease to 0 (see for instance [14]) as $t$ goes to $\infty$.

When (H4) holds, the stationary solution of (1.3) is given by

$$
\begin{cases}\bar{p}_{0}(a)=\frac{K}{\int_{0}^{+\infty} \pi(u) d u} \pi(a) & \\ \bar{p}_{i}(a)=0 & i>0\end{cases}
$$

and will be called the 'Parasite Free Equilibrium', shortly PFE.

Note that $\bar{p}_{0}(a)$ is not necessarily stable for the purely demographic equation (the 0 -th equation in (1.3) with $p_{i} \equiv 0$ for all $i \neq 0$ ). Indeed, it is well known $[14,26]$ that it will be locally asymptotically stable if and only if

(H5) There are no solutions with $\operatorname{Re} \lambda \geq 0$ of

$$
\frac{1}{\mathcal{R}} \int_{0}^{\infty} \beta(a) \pi(a) e^{-\lambda a} d a+\frac{\psi^{\prime}(K) K \mathcal{R}}{\int_{0}^{\infty} \pi(a) d a} \int_{0}^{\infty} \pi(a) e^{-\lambda a} d a=1 .
$$


Some sufficient conditions for (H5) have been found: for instance, (H5) holds for any function $\psi$, if $\pi(a)$ is a convex function [14], which is equivalent to $\mu^{\prime}(a) \leq$ $\mu^{2}(a)$.

\section{EXISTEnCE OF POSITIVE EQUILIBRIA}

We are interested in the existence of positive (or endemic) equilibria, i.e. equilibria with $\varphi>0$.

Let $\left(p_{i}(a)\right)_{i \in \mathbb{N}}$ be a stationary solution of (1.3). Then it solves

$$
\begin{cases}p_{i}^{\prime}(a)=-(\mu(a)+\varphi+i(\alpha+\sigma)) p_{i}(a)+\sigma(i+1) p_{i+1}(a)+\varphi p_{i-1}(a) & i \geq 0 \\ p_{0}(0)=L=\psi(\bar{N}) \int_{0}^{+\infty} \beta(a) \sum_{i=0}^{+\infty} p_{i}(a) \xi^{i} d a & i \geq 1, \\ p_{i}(0)=0 & \end{cases}
$$

where

$$
\bar{N}=\sum_{i=0}^{+\infty} \int_{0}^{+\infty} p_{i}(s) d s \quad \bar{P}=\sum_{i=1}^{+\infty} i \int_{0}^{+\infty} p_{i}(s) d s \quad \varphi=\frac{h \bar{P}}{c+\bar{N}}
$$

are constant.

Therefore, disregarding the implicit boundary condition involving $p_{0}(0)$, it is known (see [2] or [15]) that the population has a Poisson's distribution at each $a$,

$$
p_{i}(a)=N(a) \frac{(x(a))^{i}}{i !} e^{-x(a)}
$$

where $N(a)$ is the total population of age $a$ and $x(a)$ is the mean number of parasites carried by a host of age $a . N(a)$ and $x(a)$ are given by

$$
x(a)=\frac{\varphi}{\alpha+\sigma}\left(1-e^{-(\alpha+\sigma) a}\right) \quad \text { and } \quad N(a)=L \pi_{\varphi}(a),
$$

where

$$
\pi_{\varphi}(a)=e^{-\int_{0}^{a} \mu(u) d u-\alpha \int_{0}^{a} x(u) d u}
$$

represents the probability for an individual to survive to age $a$ (note that the dependence on $\varphi$ is hidden in $x(u)$, and that $\left.\pi_{0}(a)=\pi(a)\right)$.

Hence for $\bar{N}$ and $\bar{P}$ we obtain

$$
\begin{aligned}
\bar{N} & =\int_{0}^{+\infty} N(a) d a=\int_{0}^{+\infty} L \pi_{\varphi}(a) d a=L G(\varphi), \\
\bar{P} & =L \int_{0}^{+\infty} x(a) \pi_{\varphi}(a) d a=\frac{L \varphi}{\alpha+\sigma} R(\varphi),
\end{aligned}
$$

having set

$$
G(\varphi)=\int_{0}^{+\infty} \pi_{\varphi}(a) d a \quad \text { and } \quad R(\varphi)=\int_{0}^{+\infty}\left(1-e^{-(\sigma+\alpha) a}\right) \pi_{\varphi}(a) d a
$$


Substituting these expressions in (3.8) one gets the equation

whence

$$
\varphi=\frac{h L \varphi R(\varphi)}{(\alpha+\sigma)(c+L G(\varphi))}
$$

$$
L=L(\varphi)=\frac{c}{\frac{h R(\varphi)}{\alpha+\sigma}-G(\varphi)}
$$

which gives $L$, the density of newborn, as function of $\varphi$, for $\varphi>0$.

In order to deal with quantities that have biological meaning we require $L(\varphi)>0$. This is true as long as $\frac{h R(\varphi)}{(\alpha+\sigma) G(\varphi)}>1$. Since, as we will show below, $\frac{R(\varphi)}{G(\varphi)}$ is a decreasing function, a necessary condition for the existence of a solution of (3.7) is $\frac{h}{\alpha+\sigma} \frac{R(0)}{G(0)}>1$, i.e.

$$
\frac{h \int_{0}^{+\infty}\left(1-e^{-(\alpha+\sigma) a}\right) \pi(a) d a}{(\alpha+\sigma) \int_{0}^{+\infty} \pi(a) d a}>1
$$

Under condition (3.11) (below we will ask for a stronger one), $\frac{h R(\varphi)}{(\alpha+\sigma) G(\varphi)}>1$ in $\left[0, \varphi_{\max }\right)$ where $\varphi_{\max }$ is such that $\frac{h R\left(\varphi_{\max }\right)}{(\alpha+\sigma) G\left(\varphi_{\max }\right)}=1$, if such a value exists, $\varphi_{\max }=$ $+\infty$ otherwise.

Using the expression obtained for $L(\varphi)$, the boundary condition of (3.7)

$$
p_{0}(0)=L=\psi(\bar{N}) \int_{0}^{+\infty} \beta(a) \sum_{i=0}^{+\infty} L \pi_{\varphi}(a) \frac{(x(a))^{i}}{i !} e^{-x(a)} \xi^{i} d a
$$

becomes, for $\varphi \in\left[0, \varphi_{\max }\right)$,

$$
1=H(\varphi)
$$

where

$$
H(\varphi):=\psi(L(\varphi) G(\varphi)) S(\varphi)=\psi\left(\frac{c}{\frac{h}{\sigma+\alpha} \frac{R(\varphi)}{G(\varphi)}-1}\right) S(\varphi)
$$

with

$$
S(\varphi):=\int_{0}^{+\infty} \beta(a) \pi_{\varphi}(a) e^{-x(a)(1-\xi)} d a .
$$

We then see that each solution of (3.7) corresponds to a solution $\varphi$ of $H(\varphi)=1$ and vice versa.

The main result of this Section is the following.

Theorem 3.1. There exists one and only one positive equilibrium if and only if

$$
R_{0}:=\frac{h K}{c+K} \frac{R(0)}{(\alpha+\sigma) G(0)}=\frac{h K}{c+K} \frac{\int_{0}^{+\infty}\left(1-e^{-(\sigma+\alpha) a}\right) \pi(a) d a}{(\alpha+\sigma) \int_{0}^{+\infty} \pi(a) d a}>1 .
$$

For its proof we need a lemma. 
Lemma 3.2. If $g(a)=a-\frac{1-e^{-(\sigma+\alpha) a}}{\sigma+\alpha}, h(a)=1-e^{-(\sigma+\alpha) a}$ and $\pi_{\varphi}(a)$ is as in (3.9) then

$$
\int_{0}^{+\infty} \pi_{\varphi}(a) d a \cdot \int_{0}^{+\infty} \pi_{\varphi}(a) g(a) h(a) d a>\int_{0}^{+\infty} \pi_{\varphi}(a) g(a) d a \cdot \int_{0}^{+\infty} \pi_{\varphi}(a) h(a) d a .
$$

Proof. Let $u(a)=\pi_{\varphi}(a) h(a)$ and $v(a)=\pi_{\varphi}(a)$. We have to prove that

$$
\int_{0}^{+\infty} v(a) d a \cdot \int_{0}^{+\infty} u(a) g(a) d a>\int_{0}^{+\infty} v(a) g(a) d a \cdot \int_{0}^{+\infty} u(a) d a .
$$

By a lemma in [11], (3.16) holds if $g$ and $\frac{u}{v}$ are increasing. This is obvious from the definitions of $g$ and $h$, since $\frac{u(a)}{v(a)}=h(a)$.

of Theorem 3.1. From (3.13) we have

$$
\begin{aligned}
H^{\prime}(\varphi)=S^{\prime}(\varphi) \psi\left(\frac{c}{\frac{h}{\sigma+\alpha}\left(\frac{R(\varphi)}{G(\varphi)}-\frac{\sigma+\alpha}{h}\right)}\right)-S(\varphi) \psi^{\prime}\left(\frac{c}{\frac{h}{\sigma+\alpha}\left(\frac{R(\varphi)}{G(\varphi)}-\frac{\sigma+\alpha}{h}\right)}\right) \times \\
\quad \times \frac{c h}{\alpha+\sigma} \frac{1}{\left(\frac{h}{\alpha+\sigma} \frac{R(\varphi)}{G(\varphi)}-1\right)^{2}} \frac{d}{d \varphi}\left(\frac{R(\varphi)}{G(\varphi)}\right) .
\end{aligned}
$$

We need to compute the derivatives of the functions $G, R$ and $S$. To this aim, we will use

$$
\frac{\partial}{\partial \varphi} \pi_{\varphi}(a)=-\pi_{\varphi}(a) \frac{\alpha}{\sigma+\alpha}\left(a-\frac{1-e^{-(\sigma+\alpha) a}}{\sigma+\alpha}\right)=-\frac{\alpha}{\sigma+\alpha} \pi_{\varphi}(a) g(a),
$$

and pass the derivatives inside the integrals because

$$
\left|\frac{\partial}{\partial \varphi} \pi_{\varphi}(a)\right| \leq M a e^{-\int_{0}^{a} \mu(u) d u} \quad \text { for some } M>0
$$

for all $\varphi>0$ and the RHS is a function integrable on $(0,+\infty)$. In particular, substituting (3.18) in (3.10), and using Lemma 3.2, we obtain

$$
\begin{aligned}
R^{\prime}(\varphi) G(\varphi)-R(\varphi) G^{\prime}(\varphi)= & -\frac{\alpha}{\sigma+\alpha} \int_{0}^{+\infty} h(a) \pi_{\varphi}(a) g(a) d a \cdot \int_{0}^{+\infty} \pi_{\varphi}(a) d a \\
& +\frac{\alpha}{\sigma+\alpha} \int_{0}^{+\infty} h(a) \pi_{\varphi}(a) d a \cdot \int_{0}^{+\infty} \pi_{\varphi}(a) g(a) d a<0 .
\end{aligned}
$$

Therefore

$$
\frac{d}{d \varphi}\left(\frac{R(\varphi)}{G(\varphi)}\right)=\frac{R^{\prime}(\varphi) G(\varphi)-R(\varphi) G^{\prime}(\varphi)}{G(\varphi)^{2}}<0 .
$$

Moreover

$$
\begin{aligned}
S^{\prime}(\varphi) & =-\frac{\alpha}{\sigma+\alpha} \int_{0}^{+\infty} \beta(a) \pi_{\varphi}(a) g(a) e^{-x(a)(1-\xi)} d a \\
& -\frac{(1-\xi)}{\sigma+\alpha} \int_{0}^{+\infty} \beta(a) \pi_{\varphi}(a) h(a) e^{-x(a)(1-\xi)} d a<0 .
\end{aligned}
$$


Substituting these computations in (3.17) and remembering that $\psi^{\prime}(\cdot)<0$ by assumption (H3), we obtain $H^{\prime}(\cdot)<0$. This immediately yields uniqueness of the solutions of (3.12).

As for the existence of solutions, we find $\lim _{\varphi \rightarrow \varphi_{\max }^{-}} H(\varphi)=0$.

In fact, if $\varphi_{\max }<+\infty$, then

$$
\lim _{\varphi \rightarrow \varphi_{\max }} H(\varphi)=\lim _{x \rightarrow+\infty} \psi(x) S\left(\varphi_{\max }\right)=0,
$$

because of assumption (H3). On the other hand, if $\varphi_{\max }=+\infty$ we have $\lim _{\varphi \rightarrow+\infty} S(\varphi)=$ 0 because of Lebesgue's convergence theorem.

Therefore it follows that there exists one (and only one) $\varphi>0$ such that $H(\varphi)=$ 1 if and only if $H(0)>1$ [and (3.11) holds].

We have

$$
H(0)=\psi\left(\frac{c}{\frac{h R(0)}{(\alpha+\sigma) G(0)}-1}\right) \int_{0}^{+\infty} \beta(a) \pi(a) d a=\psi\left(\frac{c}{\frac{h R(0)}{(\alpha+\sigma) G(0)}-1}\right) \mathcal{R} .
$$

Recalling that $\psi$ is a strictly decreasing function and $\psi(K) \mathcal{R}=1$, we see that $H(0)>1$ is equivalent to

$$
\frac{c}{\frac{h R(0)}{(\alpha+\sigma) G(0)}-1}<K
$$

i.e., after some algebra, (3.15).

Note finally that (3.15) implies (3.11), so that we can dispense with the latter.

\section{Abstract SETting AND Linearization PRINCIPLE}

In order to study the stability of the PFE we follow the abstract approach already described in [23], and use semigroup theory. To perform this, we transform system (1.3) into an abstract Cauchy problem

$$
\left\{\begin{array}{l}
p^{\prime}(t)=A(p(t)+H(p(t)))+F(p(t)) \\
p(0)=p^{0}
\end{array}\right.
$$

on a suitable Banach space $X$. A natural choice for $X$ is

$$
X:=\left\{p=\left(p_{i}\right)_{i \in \mathbb{N}}: p_{i} \in L^{1}(0,+\infty) \forall i \geq 0, \sum_{i=1}^{+\infty} i \int_{0}^{+\infty}\left|p_{i}(a)\right| d a<\infty\right\}
$$

endowed with the norm

$$
\|p\|:=\int_{0}^{+\infty}\left|p_{0}(a)\right| d a+\sum_{i=1}^{+\infty} i \int_{0}^{+\infty}\left|p_{i}(a)\right| d a
$$


As for the operators $A, F$ and $H$, we let $A$ be the closure of the (closable) linear operator $\mathcal{A}$ on $X$ defined by

$$
\begin{aligned}
& D(\mathcal{A})=\left\{p \in X: p_{i} \in W^{1,1}(0,+\infty), p_{i}(0)=0 \forall i \geq 0,\right. \text { and } \\
&\text { there exists } \left.N \in \mathbb{N} \text { such that } p_{i} \equiv 0 \text { for all } i>N\right\} \\
&(\mathcal{A} p)_{i}(a):=-p_{i}^{\prime}(a)-(\mu(a)+i(\alpha+\sigma)) p_{i}(a)+(i+1) \sigma p_{i+1}(a) \quad \text { for } i \geq 0,
\end{aligned}
$$

$F$ is the non linear operator given by

$$
(F(p))_{i}=\frac{h \sum_{i=1}^{+\infty} i \int_{0}^{+\infty} p_{i}(a) d a}{c+\sum_{i=0}^{+\infty} \int_{0}^{+\infty} p_{i}(a) d a}\left(p_{i-1}-p_{i}\right), \quad i \geq 0
$$

having set $p_{-1} \equiv 0 . F$ is defined on $E$ where

$$
E:=\left\{p \in X: c+\sum_{i=0}^{+\infty} \int_{0}^{+\infty} p_{i}(s) d s \neq 0\right\} .
$$

Finally $H$, the 'multiplicative perturbation' (see [7] for more details), is:

$$
\begin{aligned}
(H p)_{0}(a) & =-\psi\left(\int_{0}^{+\infty} \sum_{i=0}^{+\infty} p_{i}(s) d s\right)\left(\int_{0}^{+\infty} \beta(s) \sum_{i=0}^{+\infty} p_{i}(s) \xi^{i} d s\right) \pi(a), \\
(H p)_{i} & \equiv 0 \quad \text { for } i \geq 1 .
\end{aligned}
$$

In this approach, the boundary condition (the second equation of (1.3) is "moved" to the RHS of (4.19)). Indeed $(p+H p) \in D(A)$ if and only if the components of $p$ are in $W^{1,1}(0,+\infty)$ and $p$ satisfies the conditions

$$
\begin{aligned}
& p_{0}(0)=\psi\left(\int_{0}^{+\infty} \sum_{i=0}^{+\infty} p_{i}(s) d s\right)\left(\int_{0}^{+\infty} \beta(s) \sum_{i=0}^{+\infty} p_{i}(s) \xi^{i} d s\right) \\
& p_{i}(0)=0 \quad \text { for } i \geq 1
\end{aligned}
$$

which are exactly the boundary conditions in (1.3).

Global existence and uniqueness of positive solutions of (4.19) have been proved in [23] following [7]. Furthermore, we have established that the problem gives rise to a nonlinear semigroup. The assumptions used are:

$\left(\mathcal{H}_{1}\right) A: D(A) \subset X \rightarrow X$ is the generator of a $C_{0}$-semigroup $e^{t A}$ on a Banach space $X$

$\left(\mathcal{H}_{2}\right) H: X \rightarrow F_{A}$ and $F: X \rightarrow X$ are locally Lipschitz continuous, i. e. for all $R>0$ there exist $L_{R}, K_{R}>0$ such that

$$
|H(p)-H(q)|_{F_{A}} \leq L_{R}\|p-q\|, \quad\|F(p)-F(q)\| \leq K_{R}\|p-q\|
$$

for all $p, q \in X$ such that $\|p\|,\|q\| \leq R$ 
(more generally, $H$ and $F$ could be defined only on an open set of $X$ ).

We have denoted by $F_{A}$ the Favard class of $A$ (see [3] for details), i.e.

$$
F_{A}=\left\{p \in X: \limsup _{t \rightarrow 0^{+}} \frac{1}{t}\left\|e^{t A} p-p\right\|<+\infty\right\}
$$

which is a Banach space with the norm

$$
|p|_{F_{A}}:=\|p\|+\limsup _{t \rightarrow 0^{+}} \frac{1}{t}\left\|e^{t A} p-p\right\| .
$$

We recall (Theorem 3.4.3 in [3]) that $F_{A}$ is equal to the interpolation space $(X, D(A))_{1, \infty ; K}$.

This theorem has been proved in [23]:

Theorem 4.1. Let $A, H, F$ satisfy $\left(\mathcal{H}_{1}\right),\left(\mathcal{H}_{2}\right)$. Then

a) for each $p^{0} \in X$ there exists a unique (local) mild solution of (4.19) i.e. a continuous function $t \rightarrow p(t)$ satisfying the integral equation

$$
p(t)=e^{t A} p^{0}+A \int_{0}^{t} e^{(t-s) A} H(p(s)) d s+\int_{0}^{t} e^{(t-s) A} F(p(s)) d s ;
$$

b) if $H$ and $F$ are continuously differentiable and $\left(p^{0}+H\left(p^{0}\right)\right) \in D(A)$ then $p(t)$ is a classical solution of (4.19), i.e. $p(t)+H(p(t)) \in D(A)$ for each $t \in\left[0, t_{\max }\right), p(t)$ is differentiable and satisfies the equation (4.19) for each $0 \leq t<t_{\max }$.

c) The mild solutions depend continuously on the initial datum and give rise to a nonlinear semigroup $T(t)$.

Moreover, when A, F and $H$ are given by (4.20), (4.21) and (4.22), and (H1)-(H4) hold, if $p^{0} \geq 0$, the solutions are global, i.e. $T(t)$ is defined for all $t \geq 0$.

A crucial property (see [6]) for this proof that we will use in the sequel is the following: if $f \in C\left([0, T] ; F_{A}\right)$ then

$$
\int_{0}^{t} e^{(t-s) A} f(s) d s \in D(A)
$$

and

$$
\left\|A \int_{0}^{t} e^{(t-s) A} f(s) d s\right\| \leq M \int_{0}^{t} e^{\omega(t-s)}|f(s)|_{F_{A}} d s
$$

for all $0 \leq t \leq T$, where $M \geq 1$ and $\omega \in \mathbb{R}$ are such that $\left\|e^{t A}\right\| \leq M e^{\omega t}$.

We are now interested in analysing the stability of the equilibria of the equation (4.19). Indeed, since solutions of (4.19) are generally to be intended in the mild sense (4.24), we give the following definition.

Definition 4.2. A point $p^{*} \in X$ is an equilibrium of the equation (4.24) if $p^{*}$ solves

$$
p^{*}=e^{t A} p^{*}+A \int_{0}^{t} e^{s A} H\left(p^{*}\right) d s+\int_{0}^{t} e^{s A} F\left(p^{*}\right) d s
$$

for each $t \geq 0$. 
Remark 4.3. Indeed $p^{*}$ is an equilibrium in the previous sense if and only if $p^{*}+H\left(p^{*}\right) \in D(A)$ and $A\left(p^{*}+H\left(p^{*}\right)\right)+F\left(p^{*}\right)=0$.

In fact, the latter clearly implies the former. Conversely, let $p^{*}$ satisfy (4.26). From

$$
p^{*}=e^{t A} p^{*}+e^{t A} H\left(p^{*}\right)-H\left(p^{*}\right)+\int_{0}^{t} e^{s A} F\left(p^{*}\right) d s,
$$

or, equivalently,

$$
\left(e^{t A}-I\right)\left(p^{*}+H\left(p^{*}\right)\right)+\int_{0}^{t} e^{s A} F\left(p^{*}\right) d s=0
$$

we obtain

$$
\frac{e^{t A}-I}{t}\left(p^{*}+H\left(p^{*}\right)\right)=-\frac{1}{t} \int_{0}^{t} e^{A s} F\left(p^{*}\right) d s
$$

The right-hand side converges to $-F\left(p^{*}\right)$ as $t \rightarrow 0^{+}$and thus the same is true for the left hand side. This means that $p^{*}+H\left(p^{*}\right) \in D(A)$ and that $A\left(p^{*}+H\left(p^{*}\right)\right)+$ $F\left(p^{*}\right)=0$.

Let now $p^{*}$ denote an equilibrium of (4.19). We wish to prove a linearization principle for the asymptotic stability or instability of the equilibrium $p^{*}$. This is well known (see, for instance, [26]) for semilinear equations of the type $u^{\prime}=$ $A u+F(u)$. More generally, in [5] a linearization theorem is proved for nonlinear semigroups; here, we show that we can apply this theorem to our case, simply linearizing $H$ and $F$.

Assume that $H: X \longrightarrow F_{A}$ and $F: E \subset X \longrightarrow X$ are $C^{1}$ and let $H^{\prime}\left(p^{*}\right)$ and $F^{\prime}\left(p^{*}\right)$ be the Fréchet derivatives of $H$ and $F$ at $p^{*}$. For $p \in X$ such that $p+H^{\prime}\left(p^{*}\right) p \in D(A)$ define

$$
B_{p^{*}} p:=A\left(I+H^{\prime}\left(p^{*}\right)\right) p+F^{\prime}\left(p^{*}\right) p .
$$

We now show that $B_{p^{*}}$ generates a $C_{0^{-}}$-semigroup which is the Fréchet derivative of the nonlinear semigroup $T(t)$.

Proposition 4.4. The linear operator $B_{p^{*}}$ defined in (4.27) generates a $C_{0}$-semigroup.

Proof. Since $H^{\prime}\left(p^{*}\right): X \rightarrow F_{A}$ is linear and continuous, the results in [4] about multiplicative perturbations can be applied and hence $A\left(I+H^{\prime}\left(p^{*}\right)\right)$ is the generator of a $C_{0}$-semigroup. Moreover, since the additive perturbation $F^{\prime}\left(p^{*}\right)$ is bounded and linear, $B_{p^{*}}$ is the generator of a $C_{0}$-semigroup $e^{t B_{p^{*}}}$ (see, for instance, [10] Ch. III).

Proposition 4.5. The operator $B_{p^{*}}$ generates the Fréchet-derivative at $p^{*}$ of the nonlinear semigroup $T(t)$, i.e. $e^{t B_{p^{*}}}$ is such that

$$
\lim _{q \rightarrow p^{*}} \frac{\left\|T(t) q-p^{*}-e^{t B_{p^{*}}}\left(q-p^{*}\right)\right\|}{\left\|q-p^{*}\right\|}=0,
$$


and the convergence is uniform for $t \in[0, T], T>0$.

Proof. Since $p^{*}$ is an equilibrium then $p\left(t, p^{*}\right):=T(t) p^{*}=p^{*}$ for all $t \geq 0$. For $q \in X$ set

$$
w_{q}^{0}:=q-p^{*}, \quad w_{q}(t)=p(t, q)-p^{*}, \quad \text { and } \quad v_{q}(t)=e^{t B_{p^{*}}} w_{q}^{0} .
$$

We have to prove that

$$
\lim _{\left\|w_{q}^{0}\right\| \rightarrow 0} \frac{\left\|w_{q}(t)-v_{q}(t)\right\|}{\left\|w_{q}^{0}\right\|}=0,
$$

uniformly for $t \in[0, T], T>0$.

Using (4.26) one sees that

$$
\begin{aligned}
\| w_{q}(t)- & v_{q}(t)\|=\| A \int_{0}^{t} e^{(t-s) A}\left[H\left(p^{*}+w_{q}(s)\right)-H\left(p^{*}\right)-H^{\prime}\left(p^{*}\right) w_{q}(s)\right] d s \\
& +A \int_{0}^{t} e^{(t-s) A} H^{\prime}\left(p^{*}\right)\left(w_{q}(s)-v_{q}(s)\right) d s \\
& +\int_{0}^{t} e^{(t-s) A}\left[F\left(p^{*}+w_{q}(s)\right)-F\left(p^{*}\right)-F^{\prime}\left(p^{*}\right) w_{q}(s)\right] d s \\
& +\int_{0}^{t} e^{(t-s) A} F^{\prime}\left(p^{*}\right)\left(w_{q}(s)-v_{q}(s)\right) d s \|
\end{aligned}
$$

and hence by $(4.25)$

$$
\begin{aligned}
\left\|w_{q}(t)-v_{q}(t)\right\| \leq & M\left(\int_{0}^{t} e^{\omega(t-s)}\left|H\left(p^{*}+w_{q}(s)\right)-H\left(p^{*}\right)-H^{\prime}\left(p^{*}\right) w_{q}(s)\right|_{F_{A}} d s\right. \\
& \left.+\int_{0}^{t} e^{\omega(t-s)} \mid H^{\prime}\left(p^{*}\right)\right)\left.\right|_{\mathcal{L}\left(X, F_{A}\right)}\left\|w_{q}(s)-v_{q}(s)\right\| d s \\
& +\int_{0}^{t} e^{\omega(t-s)}\left\|F\left(p^{*}+w_{q}(s)\right)-F\left(p^{*}\right)-F^{\prime}\left(p^{*}\right) w_{q}(s)\right\| d s \\
& \left.\left.+\int_{0}^{t} e^{\omega(t-s)} \| F^{\prime}\left(p^{*}\right)\right)\left\|_{\mathcal{L}(X)}\right\| w_{q}(s)-v_{q}(s) \| d s\right) .
\end{aligned}
$$

Now, for arbitrary $\eta>0$

$$
\left|H\left(p^{*}+w_{q}(s)\right)-H\left(p^{*}\right)-H^{\prime}\left(p^{*}\right) w_{q}(s)\right|_{F_{A}} \leq \eta\left\|w_{q}(s)\right\|
$$

provided that $\left\|w_{q}(s)\right\| \leq \varepsilon(\eta)$. Since the solutions of (4.19) depend continuously on the initial datum, it is clear that $\left\|w_{q}(s)\right\| \leq \varepsilon(\eta)$ for $0 \leq s \leq T, T>0$, if $\left\|w_{q}^{0}\right\|$ is small enough.

By the same argument

$$
\left\|F\left(p^{*}+w_{q}(s)\right)-F\left(p^{*}\right)-F^{\prime}\left(p^{*}\right) w_{q}(s)\right\| \leq \eta\left\|w_{q}(s)\right\|
$$

for $0 \leq s \leq T$ if $\left\|w_{q}(s)\right\| \leq \delta(\eta)$.

Moreover, it was shown in the proof of Theorem 2.2 in [23] that there exists $L>0$ such that

$$
\left\|w_{q}(s)\right\| \leq M e^{L T}\left\|w_{q}^{0}\right\| \quad \text { for } 0 \leq s \leq T
$$


Thus, if $\left\|w_{q}^{0}\right\|$ is small enough, we have

$$
\begin{aligned}
\left\|w_{q}(t)-v_{q}(t)\right\| \leq M \eta \int_{0}^{t} e^{\omega(t-s)}\left\|w_{q}(s)\right\| d s+M\left|H^{\prime}\left(p^{*}\right)\right| \int_{0}^{t} e^{\omega(t-s)}\left\|w_{q}(s)-v_{q}(s)\right\| d s \\
\quad+M \eta \int_{0}^{t} e^{\omega(t-s)}\left\|w_{q}(s)\right\| d s+M\left\|F^{\prime}\left(p^{*}\right)\right\| \int_{0}^{t} e^{\omega(t-s)}\left\|w_{q}(s)-v_{q}(s)\right\| d s \\
\leq \frac{2 M^{2} \eta}{|\omega|}\left\|w_{q}^{0}\right\|\left|e^{\omega T}-1\right| e^{L T} \\
\quad+M\left(\left|H^{\prime}\left(p^{*}\right)\right|+\left\|F^{\prime}\left(p^{*}\right)\right\|\right) e^{|\omega| T} \int_{0}^{t}\left\|w_{q}(s)-v_{q}(s)\right\| d s .
\end{aligned}
$$

Finally, by Gronwall Lemma it follows

$$
\left\|w_{q}(t)-v_{q}(t)\right\| \leq \frac{2 M^{2} \eta}{|\omega|}\left\|w_{q}^{0}\right\| e^{L T}\left|e^{\omega T}-1\right| e^{M e^{|\omega| T} T\left(\left|H^{\prime}\left(p^{*}\right)\right|+\left\|F^{\prime}\left(p^{*}\right)\right\|\right)} .
$$

Since $\eta$ can be taken arbitrary, the thesis is proved.

Applying the results in [5] (slightly modified for the instability clause), we then obtain the following

Corollary 4.6. If $\omega_{0}\left(B_{p^{*}}\right)<0$, then $p^{*}$ is exponentially asymptotically stable for (4.19). If $\omega_{0}\left(B_{p^{*}}\right)>0, X=X_{1} \oplus X_{2}$ with $X_{1}$ finite dimensional, $X_{i}$ invariant with respect to $e^{t B_{p *}}$ for $i=1,2$, and

$$
\min \left\{\operatorname{Re} \lambda: \lambda \in \sigma\left(\left.B_{p^{*}}\right|_{X_{1}}\right)\right\}>\max \left\{\omega_{0}\left(\left.B_{p^{*}}\right|_{X_{2}}\right), 0\right\}
$$

then $p^{*}$ is unstable for (4.19).

\section{Stability CONDitions}

In this Section we will apply the results of the linearization principle proved in the previous Section to the case where $A, F$ and $H$ are given by (4.20), (4.21) and (4.22), and the equilibrium $p^{*}$ is the PFE. In order to compute $\omega_{0}\left(B_{p^{*}}\right)$, we will use repeatedly the following general theorem. In essence it says that for a block triangular operator, one needs to compute only the growth rates of the diagonal blocks. This would be trivial without the multiplicative perturbation; in this case we have to add assumption (5.28).

Theorem 5.1. Let $A_{0}$ and $B_{1}$ be the generators of $C_{0}$-semigroups on the Banach spaces $Y_{0}$ and $Y_{1}$ respectively. Let $H_{i} \in \mathcal{L}\left(Y_{i}, F_{A_{0}}\right)$ for $i=0,1$ and $B_{10} \in \mathcal{L}\left(Y_{1}, Y_{0}\right)$. Then

(i) the operator $B$ defined by

$$
B\left(\begin{array}{c}
q_{0} \\
q_{1}
\end{array}\right)=\left(\begin{array}{c}
A_{0}\left(q_{0}+H_{0} q_{0}+H_{1} q_{1}\right)+B_{10} q_{1} \\
B_{1} q_{1}
\end{array}\right)
$$

is the generator of a $C_{0}$-semigroup on $Y=Y_{0} \oplus Y_{1}$; 
(ii) if, letting $I_{0}$ be the identity in $Y_{0}$,

$$
\left(I_{0}+H_{0}\right)^{-1} \in \mathcal{L}\left(F_{A_{0}}, F_{A_{0}\left(I_{0}+H_{0}\right)}\right)
$$

then

$$
\omega_{0}\left(B_{1}\right)<0 \quad \text { and } \quad \omega_{0}\left(A_{0}\left(I_{0}+H_{0}\right)\right)<0 \Longleftrightarrow \omega_{0}(B)<0 .
$$

Before proving the theorem we need a lemma.

Lemma 5.2. Let $A$ be the generator of a $C_{0}$-semigroup on the Banach space $X$ and suppose that $H \in \mathcal{L}\left(X, F_{A}\right),(I+H)^{-1} \in \mathcal{L}\left(F_{A}, F_{A(I+H)}\right), f \in C\left([0, T], F_{A}\right)$, $g \in C([0, T], X)$. Then the solution of

$$
v(t)=e^{t A} v^{0}+A \int_{0}^{t} e^{(t-s) A} H(v(s)) d s+A \int_{0}^{t} e^{(t-s) A} f(s) d s+\int_{0}^{t} e^{(t-s) A} g(s) d s
$$

is given by

$$
\begin{gathered}
v(t)=e^{t A(I+H)} v^{0}+A(I+H) \int_{0}^{t} e^{(t-s) A(I+H)}(I+H)^{-1} f(s) d s \\
+\int_{0}^{t} e^{(t-s) A(I+H)} g(s) d s
\end{gathered}
$$

Proof. Since, by Theorem 4.1, (5.29) has a unique solution, we have only to show that $v(t)$ defined in (5.30) solves (5.29). To begin, let $f \in C^{1}\left([0, T], F_{A}\right)$. Using in (5.30) the definition [6] of $e^{t A(I+H)}$ we get

$$
\begin{aligned}
v(t) & =e^{t A} v^{0}+A \int_{0}^{t} e^{(t-s) A} H e^{s A(I+H)} v^{0} d s \\
& +A(I+H) \int_{0}^{t} e^{(t-s) A(I+H)}(I+H)^{-1} f(s) d s+\int_{0}^{t} e^{(t-s) A(I+H)} g(s) d s .
\end{aligned}
$$

and then, substituting $e^{s A(I+H)} v^{0}$ with $v(s)$ minus the rest of the RHS of (5.30), we get

$$
\begin{aligned}
v(t)= & e^{t A} v^{0}+A \int_{0}^{t} e^{(t-s) A} H\left[v(s)-A(I+H) \int_{0}^{s} e^{(s-u) A(I+H)}(I+H)^{-1} f(u) d u\right. \\
& \left.-\int_{0}^{s} e^{(s-u) A(I+H)} g(u) d u\right] d s \\
& +A(I+H) \int_{0}^{t} e^{(t-s) A(I+H)}(I+H)^{-1} f(s) d s+\int_{0}^{t} e^{(t-s) A(I+H)} g(s) d s \\
= & v_{1}(t)+v_{2}(t)
\end{aligned}
$$


where, performing also some integration by parts,

$$
\begin{aligned}
v_{1}(t) & =e^{t A} v^{0}+A \int_{0}^{t} e^{(t-s) A} H(v(s)) d s-A \int_{0}^{t} e^{(t-s) A} H\left[e^{s A(I+H)}(I+H)^{-1} f(0)\right. \\
& \left.-(I+H)^{-1} f(s)+\int_{0}^{s} e^{(s-u) A(I+H)}(I+H)^{-1} f^{\prime}(u) d u\right] d s \\
v_{2}(t) & =e^{t A(I+H)}(I+H)^{-1} f(0)-(I+H)^{-1} f(t)+\int_{0}^{t} e^{(t-s) A(I+H)}(I+H)^{-1} f^{\prime}(s) d s \\
& -A \int_{0}^{t} e^{(t-s) A} H \int_{0}^{s} e^{(s-u) A(I+H)} g(u) d u d s+\int_{0}^{t} e^{(t-s) A(I+H)} g(s) d s .
\end{aligned}
$$

Now, using the definition of $e^{t A(I+H)}$ in $v_{2}(t)$, we get

$$
\begin{aligned}
v_{2}(t)= & e^{t A}(I+H)^{-1} f(0)+A \int_{0}^{t} e^{(t-s) A} H e^{s A(I+H)}(I+H)^{-1} f(0) d s \\
& -(I+H)^{-1} f(t)+\int_{0}^{t} e^{(t-s) A}(I+H)^{-1} f^{\prime}(s) d s \\
& +\int_{0}^{t} A \int_{0}^{t-s} e^{(t-s-u) A} H e^{u A(I+H)} d u(I+H)^{-1} f^{\prime}(s) d s \\
& -A \int_{0}^{t} e^{(t-s) A} H \int_{0}^{s} e^{(s-u) A(I+H)} g(u) d u d s+\int_{0}^{t} e^{(t-s) A} g(s) d s \\
& +\int_{0}^{t} A \int_{0}^{t-s} e^{(t-s-u) A} H e^{u A(I+H)} g(s) d u d s .
\end{aligned}
$$

Cancelling the terms in $v_{1}(t)$ and $v_{2}(t)$ with opposite signs, we get

$$
\begin{aligned}
v(t)= & e^{t A} v^{0}+A \int_{0}^{t} e^{(t-s) A} H(v(s)) d s+e^{t A}(I+H)^{-1} f(0)-(I+H)^{-1} f(t) \\
& +\int_{0}^{t} e^{(t-s) A}(I+H)^{-1} f^{\prime}(s) d s \\
& +A \int_{0}^{t} e^{(t-s) A} H(I+H)^{-1} f(s) d s+\int_{0}^{t} e^{(t-s) A} g(s) d s
\end{aligned}
$$

which becomes, via an integration by parts,

$$
\begin{aligned}
v(t)= & e^{t A} v^{0}+A \int_{0}^{t} e^{(t-s) A} H(v(s)) d s+A \int_{0}^{t} e^{(t-s) A}(I+H)^{-1} f(s) d s \\
& +A \int_{0}^{t} e^{(t-s) A} H(I+H)^{-1} f(s) d s+\int_{0}^{t} e^{(t-s) A} g(s) d s \\
= & e^{t A} v^{0}+A \int_{0}^{t} e^{(t-s) A} H(v(s)) d s+A \int_{0}^{t} e^{(t-s) A} f(s) d s+\int_{0}^{t} e^{(t-s) A} g(s) d s
\end{aligned}
$$

which is the thesis.

Using a density argument, the same can be proved when $f \in C\left([0, T], F_{A}\right)$. 
of Theorem 5.1. Consider the operator

$$
\widetilde{B}=\left(\begin{array}{cc}
A_{0} & 0 \\
0 & B_{1}
\end{array}\right)
$$

It clearly generates a $C_{0}$-semigroup on $Y=Y_{0} \oplus Y_{1}$ while

$$
H\left(\begin{array}{l}
q_{0} \\
q_{1}
\end{array}\right)=\left(\begin{array}{c}
H_{0} q_{0}+H_{1} q_{1} \\
0
\end{array}\right)
$$

defines an operator $H \in \mathcal{L}\left(X, F_{\widetilde{B}}\right)$. Now a straightforward application of the perturbation theorem by Desch and Schappacher [4] and then the classical result about bounded perturbations prove the first statement.

About $(i i)$, if $\omega_{0}\left(B_{1}\right) \geq 0$ or $\omega_{0}\left(A_{0}\left(I_{0}+H_{0}\right)\right) \geq 0$ it is clear that $\omega_{0}(B) \geq 0$ : it suffices to apply $e^{t B}$ to $\left(0, q_{1}\right)^{T}$ or $\left(q_{0}, 0\right)^{T}$.

Suppose now that $\omega_{0}\left(B_{1}\right)<0$ and $\omega_{0}\left(A_{0}\left(I_{0}+H_{0}\right)\right)<0$. There exist constants $M, \eta>0$ such that

$$
\left\|e^{t B_{1}}\right\| \leq M e^{-\eta t}, \quad\left\|e^{t A_{0}\left(I_{0}+H_{0}\right)}\right\| \leq M e^{-\eta t} .
$$

Set

$$
\left(\begin{array}{l}
q_{0}(t) \\
q_{1}(t)
\end{array}\right)=e^{t B}\left(\begin{array}{l}
q_{0}^{0} \\
q_{1}^{0}
\end{array}\right)
$$

We have $q_{1}(t)=e^{t B_{1}} q_{1}^{0}$ while $q_{0}(t)$ solves

$$
\begin{aligned}
q_{0}(t)= & e^{t A_{0}} q_{0}^{0}+A_{0} \int_{0}^{t} e^{(t-s) A_{0}} H_{0}\left(q_{0}(s) d s\right. \\
& +A_{0} \int_{0}^{t} e^{(t-s) A_{0}} H_{1}\left(q_{1}(s)\right) d s+\int_{0}^{t} e^{(t-s) A_{0}} B_{10} q_{1}(s) d s .
\end{aligned}
$$

Apply the previous lemma with $f(t)=H_{1} q_{1}(t)$ and $g(t)=B_{10} q_{1}(t)$. Hence

$$
\begin{aligned}
q_{0}(t)= & e^{t A_{0}\left(I_{0}+H_{0}\right)} q_{0}+\int_{0}^{t} e^{(t-s) A_{0}\left(I_{0}+H_{0}\right)} B_{10}\left(q_{1}(s)\right) d s \\
& +A_{0}\left(I_{0}+H_{0}\right) \int_{0}^{t} e^{(t-s) A_{0}\left(I_{0}+H_{0}\right)}\left(I_{0}+H_{0}\right)^{-1} H_{1}\left(q_{1}(s)\right) d s .
\end{aligned}
$$

From $\left\|q_{1}(t)\right\| \leq M e^{-\eta t}\left\|q_{1}^{0}\right\|$ it follows that

$$
\begin{aligned}
\left\|q_{0}(t)\right\| \leq & M e^{-\eta t}\left\|q_{0}^{0}\right\|+M^{2} \int_{0}^{t} e^{-\eta(t-s)}\left\|B_{10}\right\|_{\mathcal{L}\left(X_{1}, X_{0}\right)} e^{-\eta s}\left\|q_{1}^{1}\right\| d s \\
& +M^{2} \int_{0}^{t} e^{-\eta(t-s)}\left\|\left(I_{0}+H_{0}\right)^{-1}\right\|_{\mathcal{L}\left(F_{A_{0}}, F_{A_{0}\left(I_{0}+H_{0}\right)}\right)}\left\|H_{1}\right\|_{\mathcal{L}\left(X_{1}, F_{A_{0}}\right)} e^{-\eta s}\left\|q_{1}^{0}\right\| d s \\
\leq & e^{-\eta t}\left(M\left\|q_{0}^{0}\right\|+M^{2}\left(\left\|\left(I_{0}+H_{0}\right)^{-1}\right\|\left\|H_{1}\right\|+\left\|B_{10}\right\|\right) t\left\|q_{1}^{1}\right\|\right) .
\end{aligned}
$$

Thus the second statement is proved. 
We are now going to show that Theorem 5.1 can be applied to $B=B_{\bar{p}}$. If now $A, F, H$ are the operators defined in $(4.20),(4.21),(4.22)$ and $p^{*}=\bar{p}$, that is the $\mathrm{PFE}$, we have, recalling (2.5) and (2.6),

$$
\begin{aligned}
& {\left[H^{\prime}(\bar{p}) u\right]_{0}(a)=-\left(\frac{\psi^{\prime}(K) K \mathcal{R}}{\int_{0}^{+\infty} \pi(s) d s} \sum_{i=0}^{+\infty} \int_{0}^{+\infty} u_{i}(s) d s+\frac{1}{\mathcal{R}} \int_{0}^{+\infty} \beta(s) \sum_{i=0}^{+\infty} u_{i}(s) \xi^{i} d s\right) \pi(a)} \\
& {\left[H^{\prime}(\bar{p}) u\right]_{i}(a)=0 \quad \text { if } \quad i>0} \\
& {\left[F^{\prime}(\bar{p}) u\right]_{0}(a)=-\left[F^{\prime}(\bar{p}) u\right]_{1}(a)=-\frac{h K}{c+K} \frac{\pi(a)}{\int_{0}^{+\infty} \pi(s) d s} \sum_{i=0}^{+\infty} i \int_{0}^{+\infty} u_{i}(s) d s} \\
& {\left[F^{\prime}(\bar{p}) u\right]_{i}(a)=0 \quad \text { if } \quad i>1 .}
\end{aligned}
$$

Set

$$
X=X_{0} \oplus \bar{X}_{1}, \quad \bar{X}_{1}=X_{1} \oplus \bar{X}_{2}
$$

where

$$
\begin{gathered}
X_{0}=X_{1}=L^{1}(0,+\infty) \\
\bar{X}_{2}=\left\{\bar{q}_{2}=\left(q_{i}\right)_{i \geq 2}: q_{i} \in L^{1}(0,+\infty), \sum_{i=2}^{+\infty} \int_{0}^{+\infty} i\left|q_{i}(a)\right| d a<\infty\right\}
\end{gathered}
$$

and therefore $\bar{X}_{1}=\left\{\bar{q}_{1}=\left(q_{i}\right)_{i \geq 1}: q_{i} \in L^{1}(0,+\infty), \sum_{i=1}^{+\infty} \int_{0}^{+\infty} i\left|q_{i}(a)\right| d a<\infty\right\}$.

The operator $B$ can be represented as follows:

$$
B\left(\begin{array}{c}
q_{0} \\
\bar{q}_{1}
\end{array}\right)=\left(\begin{array}{c}
A_{0}\left(q_{0}+H_{0} q_{0}+H_{1} \bar{q}_{1}\right)+B_{10} \bar{q}_{1} \\
\bar{B}_{1} \bar{q}_{1}
\end{array}\right)
$$

where

$$
\begin{gathered}
A_{0}: D\left(A_{0}\right)=\left\{q_{0} \in X_{0}: q_{0}(0)=0\right\} \longrightarrow X_{0} \\
\left(A_{0} q_{0}\right)(a)=-q_{0}^{\prime}(a)-\mu(a) q_{0}(a), \\
H_{0}: X_{0} \longrightarrow F_{A_{0}}, \quad H_{1}: \bar{X}_{1} \longrightarrow F_{A_{0}} \\
\left(H_{0} q_{0}\right)(a)=-\left(\frac{\psi^{\prime}(K) K \mathcal{R}}{\int_{0}^{+\infty} \pi(s) d s} \int_{0}^{+\infty} q_{0}(s) d s+\frac{1}{\mathcal{R}} \int_{0}^{+\infty} \beta(s) q_{0}(s) d s\right) \pi(a), \\
\left(H_{1} \bar{q}_{1}\right)(a)=-\left(\frac{\psi^{\prime}(K) K \mathcal{R}}{\int_{0}^{+\infty} \pi(s) d s} \sum_{i=1}^{+\infty} \int_{0}^{+\infty} q_{i}(s) d s+\frac{1}{\mathcal{R}} \int_{0}^{+\infty} \beta(s) \sum_{i=1}^{+\infty} q_{i}(s) \xi^{i} d s\right) \pi(a), \\
\left(B_{10} \bar{q}_{1}\right)(a)=-\frac{h X_{i=1}^{+\infty} \int_{0}^{+\infty} q_{i}(s) d s}{c+K} \bar{p}_{0}(a)+\sigma q_{1}(a),
\end{gathered}
$$

$\bar{B}_{1}$ is the closure of

$$
B_{1}: D\left(B_{1}\right) \longrightarrow \overline{X_{1}}
$$




$$
\begin{aligned}
\left(B_{1} \bar{q}_{1}\right)_{1}(a) & =-q_{1}^{\prime}(a)-(\mu(a)+\sigma+\alpha) q_{1}(a) \\
& +\frac{h}{c+K}\left(\sum_{i=1}^{+\infty} \int_{0}^{+\infty} i q_{i}(s) d s\right) \bar{p}_{0}(a)+2 \sigma q_{2}(a) \\
\left(B_{1} \bar{q}_{1}\right)_{i}(a) & =-q_{i}^{\prime}(a)-(\mu(a)+\sigma+\alpha) q_{i}(a)+(i+1) \sigma q_{i+1}(a) \quad \text { for } i>1
\end{aligned}
$$

with

$$
\begin{aligned}
D\left(B_{1}\right)=\{ & \left\{\bar{q}_{1} \in \bar{X}_{1}: q_{i} \in W^{1,1}(0,+\infty), q_{i}(0)=0 \forall i \geq 1,\right. \text { and } \\
& \text { there exists } \left.N \in \mathbb{N} \text { such that } q_{i} \equiv 0 \text { for all } i>N\right\} .
\end{aligned}
$$

One can immediately verify that $H_{0}$ and $H_{1}$ take values in $F_{A_{0}}$ and that $B_{10}$ is a bounded operator.

We also note that $B_{1}$ can be written as

$$
B_{1}\left(\begin{array}{c}
q_{1} \\
\bar{q}_{2}
\end{array}\right)=\left(\begin{array}{c}
B_{11} q_{1}+B_{21} \bar{q}_{2} \\
B_{2} \bar{q}_{2}
\end{array}\right)
$$

where

$$
\left(B_{11} q_{1}\right)(a):=-q_{1}^{\prime}(a)-(\mu(a)+\sigma+\alpha) q_{1}(a)+\frac{h}{c+K} \bar{p}_{0}(a) \int_{0}^{+\infty} q_{1}(s) d s
$$

with domain

$$
\begin{gathered}
D\left(B_{11}\right)=\left\{q_{1} \in W^{1,1}(0,+\infty): q_{1}(0)=0\right\} \\
\left(B_{2} \bar{q}_{2}\right)_{i}(a)=-q_{i}^{\prime}(a)-(\mu(a)+i(\alpha+\sigma)) q_{i}(a)+\sigma(i+1) q_{i+1}(a),
\end{gathered}
$$

with domain

$$
\begin{aligned}
& D\left(B_{2}\right)=\left\{\bar{q}_{2}=\left(q_{i}\right)_{i \geq 2}: q_{i} \in W^{1,1}(0,+\infty), q_{i}(0)=0 \text { for each } i \geq 2\right. \\
&\text { and there exists } \left.N \text { such that } q_{i} \equiv 0 \text { for all } i>N\right\},
\end{aligned}
$$

and

$$
\left(B_{21} \bar{q}_{2}\right)(a)=\frac{h \sum_{i=2}^{+\infty} i \int_{0}^{+\infty} q_{i}(s) d s}{c+K} \bar{p}_{0}(a)+2 \sigma q_{2}(a) .
$$

Proposition 5.3. The closure of $B_{1}$ generates a $C_{0}$-semigroup on $\bar{X}_{1}$.

Proof. The closure of the operator $B_{11} \oplus B_{2}$ generates a $C_{0}$-semigroup on $\bar{X}_{1}$ (as for $B_{2}$, see the proof given for the operator $A$ in [23]). Since $B_{21}$ gives rise clearly to a bounded perturbation, the classical result on bounded perturbations can be applied and the proof is achieved.

To apply Theorem 5.1 to our case we have to prove that the operators defined above satisfy the assumption (5.28). Indeed, we have:

Proposition 5.4. $I_{0}+H_{0}$ is invertible in $X_{0}$ and $\left(I_{0}+H_{0}\right)^{-1}$ belongs to $\mathcal{L}\left(X_{0}\right)$ and $\mathcal{L}\left(F_{A_{0}}, F_{A_{0}\left(I_{0}+H_{0}\right)}\right)$. 
Proof. $H_{0}$ can be rewritten as

$$
\begin{aligned}
\left(H_{0} u_{0}\right)(a) & =-\left(\psi^{\prime}(K) \frac{\mathcal{R} K}{\int_{0}^{+\infty} \pi(a) d a} \int_{0}^{+\infty} u_{0}(s) d s+\frac{1}{\mathcal{R}} \int_{0}^{+\infty} \beta(s) u_{0}(s) d s\right) \pi(a) \\
& =C\left(u_{0}\right) \pi(a)
\end{aligned}
$$

where

$$
C\left(u_{0}\right):=-\left(\psi^{\prime}(K) \frac{\mathcal{R} K}{\int_{0}^{+\infty} \pi(a) d a} \int_{0}^{+\infty} u_{0}(s) d s+\frac{1}{\mathcal{R}} \int_{0}^{+\infty} \beta(s) u_{0}(s) d s\right) .
$$

Let $v \in X_{0}$. If $q_{0}+H_{0} q_{0}=v$ then

$$
q_{0}(a)=v(a)-C\left(q_{0}\right) \pi(a) .
$$

Setting $S:=C\left(q_{0}\right), S$ must then satisfy $S=C(v-S \pi)$. Hence

$S=-\left(\psi^{\prime}(K) \frac{\mathcal{R} K}{\int_{0}^{+\infty} \pi(a) d a} \int_{0}^{+\infty}(v(a)-S \pi(a)) d a+\frac{1}{\mathcal{R}} \int_{0}^{+\infty} \beta(a)(v(a)-S \pi(a) d a)\right.$ i.e.

$$
S=\frac{\int_{0}^{+\infty} v(a) d a}{\int_{0}^{+\infty} \pi(a) d a}+\frac{\int_{0}^{+\infty} \beta(a) v(a) d a}{\mathcal{R}^{2} \psi^{\prime}(K) K} .
$$

Substituting this expression in (5.35) we obtain a unique expression for $q_{0}$. It is also easy to verify, retracing back the steps, that such a $q_{0}$ satisfies $q_{0}+H_{0} q_{0}=v$, i.e. $q_{0}=\left(I_{0}+H_{0}\right)^{-1} v$. We also easily obtain

$$
\left\|q_{0}\right\|_{X_{0}} \leq\left(2+\frac{\|\beta\|_{\infty} \int_{0}^{\infty} \pi(a) d a}{\mathcal{R}^{2}\left|\psi^{\prime}(K)\right| K}\right)\|v\|_{X_{0}} .
$$

We also have $\left(I_{0}+H_{0}\right)^{-1} \in \mathcal{L}\left(D\left(A_{0}\right), D\left(A_{0}\left(I_{0}+H_{0}\right)\right)\right)$, where $D\left(A_{0}\right)$ and $D\left(A_{0}\left(I_{0}+\right.\right.$ $\left.\left.H_{0}\right)\right)$ are Banach spaces with the graph norm. Indeed,

$$
x \in D\left(A_{0}\right) \Longleftrightarrow\left(I_{0}+H_{0}\right)^{-1} x \in D\left(A_{0}\left(I_{0}+H_{0}\right)\right)
$$

and for $x \in D\left(A_{0}\right)$

$$
\begin{aligned}
\left\|\left(I_{0}+H_{0}\right)^{-1} x\right\|_{D\left(A_{0}\left(I_{0}+H_{0}\right)\right)} & =\left\|\left(I_{0}+H_{0}\right)^{-1} x\right\|_{X_{0}}+\left\|A_{0}\left(I_{0}+H_{0}\right)\left(I_{0}+H_{0}\right)^{-1} x\right\|_{X_{0}} \\
& \leq\left\|\left(I_{0}+H_{0}\right)^{-1}\right\|_{\mathcal{L}\left(X_{0}\right)}\|x\|_{X_{0}}+\left\|A_{0} x\right\|_{X_{0}} \\
& \leq \max \left\{\left\|\left(I_{0}+H_{0}\right)^{-1}\right\|_{\mathcal{L}\left(X_{0}\right)}, 1\right\}\left(\|x\|_{X_{0}}+\left\|A_{0} x\right\|_{X_{0}}\right) \\
& =\max \left\{\left\|\left(I_{0}+H_{0}\right)^{-1}\right\|_{\mathcal{L}\left(X_{0}\right)}, 1\right\}\|x\|_{D\left(A_{0}\right)} .
\end{aligned}
$$

This proves that $\left(I_{0}+H_{0}\right)^{-1}$ is continuous as an operator from $D\left(A_{0}\right)$ to $D\left(A_{0}\left(I_{0}+\right.\right.$ $\left.H_{0}\right)$ ).

Hence, we can apply Theorem 3.2.23 of [3] and obtain that $\left(I_{0}+H_{0}\right)^{-1}$ is continuous from $\left(X_{0}, D\left(A_{0}\right)\right)_{1, \infty ; K}$ to $\left(X_{0}, D\left(A_{0}\left(I_{0}+H_{0}\right)\right)\right)_{1, \infty ; K}$ which are exactly the Favard classes stated in the thesis.

Now, the main result on the growth rate of $B$ is an easy consequence of the previous theorems and propositions. 
Proposition 5.5. For the operator $B=B_{\bar{p}}$ it holds:

$$
\omega_{0}(B)<0 \Longleftrightarrow \omega_{0}\left(B_{11}\right)<0 .
$$

Proof. Theorem 5.1 can be applied to the operator $B$ taking $Y_{0}=X_{0}$ and $Y_{1}=\bar{X}_{1}$.

Moreover, we have $\omega_{0}\left(A_{0}\left(I_{0}+H_{0}\right)\right)<0$. To prove this, we recall some facts (see [26] for details). If $A$ is a generator of a $C_{0}$-semigroup $T(t)$ and

$$
\omega_{1}(A):=\lim _{t \rightarrow+\infty} t^{-1} \log (\alpha[T(t)]),
$$

where $\alpha$ is the measure of noncompactness, then

$$
\omega_{0}(A)=\max \left\{\omega_{1}(A), \sup _{\lambda \in \sigma(A)} \mathcal{R} e(\lambda)\right\}
$$

Now, $A_{0}\left(I_{0}+H_{0}\right)$ is a linear operator of age-dependent population, widely studied in [26]. Since the required hypotheses on the fertility and death rates are satisfied (assumption (H1) corresponds to (4.68) of [26]), Theorem 4.6 of [26] can be applied to the operator $A_{0}\left(I_{0}+H_{0}\right)$ ensuring that $\omega_{1}\left(A_{0}\left(I_{0}+H_{0}\right) \leq-\mu_{-}\right.$. Hence,

$$
\omega_{0}\left(A_{0}\left(I_{0}+H_{0}\right)\right)<0 \Longleftrightarrow \sup _{\lambda \in \sigma\left(A_{0}\left(I_{0}+H_{0}\right)\right)} \mathcal{R} e(\lambda)<0
$$

where the latter is indeed a maximum.

If $\lambda \in \sigma\left(A_{0}\left(I_{0}+H_{0}\right)\right), \mathcal{R} e \lambda>-\mu_{-}$then, by Theorems 4.7 and 4.6 of [26], $\lambda \in \sigma_{P}\left(A_{0}\left(I_{0}+H_{0}\right)\right)$, that is $\lambda$ is an eigenvalue and, because of (H5), $\mathcal{R} e \lambda<0$ (in fact, the equation appearing in (H5) is precisely the characteristic equation of $\left.A_{0}\left(I_{0}+H_{0}\right)\right)$.

From Theorem 5.1 it follows that $\omega_{0}(B)<0$ if and only if $\omega_{0}\left(B_{1}\right)<0$. Furthermore, we can apply the same theorem to $B_{1}$, setting $H_{0}=0, H_{1}=0, A_{0}=B_{11}$, $B_{10}=B_{21}$ and $B_{1}=B_{2}$. Now, it is easy to show, repeating exactly the proof given for $A$ in [23], that $\omega_{0}\left(B_{2}\right)<0$. In this way, we obtain that

$$
\omega_{0}\left(B_{1}\right)<0 \Longleftrightarrow \omega_{0}\left(B_{11}\right)<0
$$

and we have the thesis.

Proposition 5.6. It holds:

$$
\omega_{0}\left(B_{11}\right)<0 \Longleftrightarrow R_{0}:=\frac{h K}{c+K} \frac{\int_{0}^{+\infty} e^{-(\sigma+\alpha) u} \int_{0}^{+\infty} \pi(s+u) d s d u}{\int_{0}^{+\infty} \pi(s) d s}<1
$$

Proof. Note that we can write

$$
B_{11}=S+T
$$

where

$$
(S q)(a):=-q^{\prime}(a)-(\mu(a)+\sigma+\alpha) q(a)
$$

with $D(S)=D\left(B_{11}\right)$, and

$$
(T q)(a)=\frac{h}{c+K} \bar{p}_{0}(a) \int_{0}^{+\infty} q(s) d s .
$$


$T$ is a compact operator in $X_{1}$, while $S$ is again an operator of age-dependent population. From Theorem 4.6 of [26] we have $\omega_{1}(S) \leq-\left(\mu_{-}+\alpha+\sigma\right)$. Moreover, since $T$ is compact, we have (Proposition 4.14 of [26])

$$
\omega_{1}(S+T)=\omega_{1}(S) .
$$

Summing up, we have obtained

$$
\omega_{0}\left(B_{11}\right)<0 \Longleftrightarrow \sup _{\lambda \in \sigma\left(B_{11}\right)} \mathcal{R} e(\lambda)<0 .
$$

First of all, let us look for eigenvalues of $B_{11}$. Let $\lambda \in \mathbb{C}$ and look for $q \in D\left(B_{11}\right)$, $q \neq 0$, such that

$$
\left(B_{11}-\lambda\right) q=0
$$

Solving this explicitly we obtain

$$
G(\lambda)=1
$$

where

$$
G(\lambda):=\frac{h}{c+K} \int_{0}^{+\infty} \int_{0}^{a} \bar{p}_{0}(s) e^{-\int_{s}^{a} \mu(\tau) d \tau} e^{-(\alpha+\sigma+\lambda)(a-s)} d s d a
$$

If $G(\lambda) \neq 1, \mathcal{R} e(\lambda)>-\left(\mu_{-}+\alpha+\sigma\right)$, and $p \in X_{1}$, we can obtain $q=\left(B_{11}-\lambda\right)^{-1}(p)$ as

$$
q(a)=\int_{0}^{a}\left[\frac{h M}{c+K} \bar{p}_{0}(s)-p(s)\right] \frac{\pi(a)}{\pi(s)} e^{-(\sigma+\alpha+\lambda)(a-s)} d s
$$

with

$$
M=-\frac{\int_{0}^{+\infty} \int_{0}^{a} p(s) \frac{\pi(a)}{\pi(s)} e^{-(\alpha+\sigma+\lambda)(a-s)} d s d a}{1-G(\lambda)} .
$$

Hence, the spectrum of $B_{11}$ in $\left\{\mathcal{R} e(\lambda)>-\left(\mu_{-}+\alpha+\sigma\right)\right\}$ reduces to the solutions of

$$
G(\lambda)=1 .
$$

Since $G(\lambda)$ is the Laplace transform of a nonnegative function, the following facts can be easily obtained using the arguments in the proof of Theorem 1.5.1 of [14]:

- there exists at most one real root $\lambda_{0}>-\left(\mu_{-}+\alpha+\sigma\right)$ of (5.39);

- if $\lambda_{0}$ exists, all the other roots $\lambda$ satisfy $\operatorname{Re} \lambda<\lambda_{0}$; if there is no real root, there are no complex roots in $\left\{\operatorname{Re} \lambda>-\left(\alpha+\sigma+\mu_{-}\right)\right\}$;

- in any strip $\{a \leq \operatorname{Re} \lambda \leq b\}$ there is at most a finite number of roots;

- if $R_{0}=G(0)>[=] 1$, then $\lambda_{0}$ exists and $\lambda_{0}>[=] 0$; on the other hand, if $R_{0}<1$, if there is a real root $\lambda_{0}$, it satisfies $\lambda_{0}<0$.

We then obtain $\sup _{\lambda \in \sigma\left(B_{11}\right)} \mathcal{R} e(\lambda)<0$ if and only if $R_{0}<1$, which, thanks to (5.37), is the thesis.

Theorem 5.7. If $R_{0}<1$, the Parasite Free Equilibrium is exponentially asymptotically stable. 
Proof. It follows straightforwardly from Propositions 5.5 and 5.6 and Corollary 4.6.

The final result is:

Theorem 5.8. If $R_{0}>1$, the Parasite Free Equilibrium is unstable.

Proof. Again, it is sufficient to apply Corollary 4.6. Indeed, let $X=Z_{1} \oplus Z_{2}$ be the spectral decomposition (see, for instance, Proposition 4.8 in [26]) relative to $\sigma(B)=\left\{\lambda_{0}\right\} \cup\left\{\lambda_{0}\right\}^{C}$. Note that, since $G^{\prime}\left(\lambda_{0}\right)<0$, one can immediately see from (5.38) that $\lambda_{0}$ is a first-order pole of $(B-\lambda I)^{-1}$, hence $Z_{1}$ is one-dimensional. Moreover, $\omega_{0}\left(\left.B\right|_{Z_{2}}\right)<\lambda_{0}$, as required and we can apply Corollary 4.6.

\section{Discussion}

We have found in the previous sections that $R_{0}>1$ is the threshold condition for this model: when $R_{0}>1$ the parasite-free equilibrium is unstable and there exists a (unique) positive equlibrium. When $R_{0}<1$ there are no positive equilibria and, if the parasite-free equilibrium is asymptotically stable for the purely demographic equation (assumption (H5)), then it is stable also for the complete system.

It is then worth trying to give a biological interpretation to the condition. We found in (3.15)

$$
R_{0}=\frac{h K}{c+K} \frac{\int_{0}^{+\infty}\left(1-e^{-(\sigma+\alpha) a}\right) \pi(a) d a}{(\alpha+\sigma) \int_{0}^{+\infty} \pi(a) d a} .
$$

Using the identity $\frac{1-e^{-(\alpha+\sigma) a}}{\alpha+\sigma}=\int_{0}^{a} e^{-(\alpha+\sigma) u} d u$ and then interchanging the order of integration, we can write

$$
R_{0}=\frac{h K}{c+K} \int_{0}^{+\infty}\left(\frac{\pi(a)}{\int_{0}^{+\infty} \pi(u) d u} \cdot \int_{0}^{+\infty} e^{-(\sigma+\alpha) s} \frac{\pi(s+a)}{\pi(a)} d s\right) d a .
$$

The factor in the inner integral represents the probability that a parasite that has infected a host of age $a$ will be alive $s$ time afterwards (when the host has age $a+s$ ). Hence, the inner integral is the expected life of a parasite that has just infected a host of age $a$.

On the other hand, the factor in the outer integral represents the probability density that a randomly chosen host (at the PFE) is of age $a$; averaging, with this weight, over all ages $a$, the whole integral gives the average life-time of a parasite in a randomly chosen host. Finally, because of the expression of the infection rate $\varphi$, $\frac{h K}{c+K}$ gives the rate at which one parasite produces new infections when introduced in a host population at its parasite-free equilibrium.

Therefore the fact that the PFE is unstable when $R_{0}>1$ means that the parasites can establish into the host population if a single parasite produces more than one infected host during its life. 
If $\mu(a) \equiv \mu$ (constant), (3.15) becomes

$$
\frac{h K}{c+K} \cdot \frac{1}{\mu+\alpha+\sigma}>1
$$

which is particularly easy to interpret. Indeed, this is exactly the condition found for parasite establishment in the simplest low-dimensional approximations $[1,19$, 22 .

Note that different patterns were found in low-dimensional systems according to the kind of approximation used [19, 25]. When approximating the parasite distribution with a negative binomial of fixed aggregation parameter $\kappa$, the threshold condition was always (6.40), independently of the value of $\kappa$. On the other hand, when using a negative binomial with variable aggregation, the threshold condition is (6.40) only when infections occur only with a single parasite (as considered here), a case that gives rise to very little aggregation. If one assumes "clumped infections" (a host gets infected with a "parcel" of larvae in the same time), it was found [22] that the value of $R_{0}$ decreases when aggregation increases.

It would therefore be interesting to study the stability of the parasite-free equilibrium in this model under the assumption of "clumped infections". The linearization theorem could be applied as well, but the technical difficulty in studying the spectrum of the linearized operator would be much higher, however, since the operator $F^{\prime}(\bar{p})$ would have all components different from 0 , and one could not exploit the block triangular structure of (5.31). It seems however possible that one can arrive at a threshold condition of the type $R_{0}>1$ where $R_{0}$ represents the average number of infecting 'parcels' produced by one 'parcel' as was used in [20].

We proved here that for $R_{0}>1$ there exists a positive equilibrium but we did not state anything about its stability. From numerical simulations [25] it appears that, when $\xi=1$, the positive equilibrium is globally attracting for all values of $R_{0}>1$, while for $0<\xi<1$ it is possible to find attracting periodic solutions. This has been indeed proved for the low-dimensional approximations [1, 22], but we seem to be very far from a conclusion for the system considered here. Local asymptotic stability of the positive equilibrium could be again studied through the linearization principle, but locating the roots of the resulting characteristic equation seems hopeless. On the other hand, using abstract bifurcation theorems, it can probably be proved that the positive equilibrium inherits the stability of the parasite-free equilibrium: namely that, if $(\mathrm{H} 5)$ is satisfied and $R_{0}>1$ but small, the positive equilibrium is stable.

One may ask what happens when (H5) is not satisfied. Then, In that case, the purely demographic equation would generally have an attracting periodic solution [14], although more complex behaviours cannot be excluded. One can probably extend the techniques used here to cover the stability of periodic solutions (see [5]) and write the condition for the stability of the parasite-free periodic solution in terms of the average (over the cycle) number of infecting larvae produced by an adult parasite being less than 1 . It seems however unlikely that one arrives at 
an explicit condition, since there are no known explicit expressions for the purely demographic cycles.

The functions used in (1.3) to describe age- and density-dependence have been chosen merely for illustrative purposes; one may have more complex expressions for the fertility rates:

$$
\beta\left(a, S_{1}(t), S_{2}(t), \ldots, S_{n}(t)\right)
$$

and analogously for $\mu$, where $S_{k}(t)$ are suitably weighted integrals of $p(a, t)$ (see [14]); the techniques would remain the same, although it is possible that, if $\mu$ depends on population size, the computations of Section 3 about positive equilibria would yield somewhat different results.

In this regard, it must be remarked that the results of Section 3 are rather unexpected. Indeed, Kretzschmar [18] found that, when there is no density-dependence, the bifurcation structure is different when $\xi=1$ and when $\xi<1$. Assuming $c=0$ in (1.2), she studied the bifurcations from the parasite-free exponential solutions of $(1.1)$ :

$$
p_{0}(t)=k e^{(\beta-\mu) t} ; \quad p_{i}(t)=0 \text { for } i \geq 1 ;
$$

for $\xi=1$ (no effect of parasites on host fertility), the branching of an exponential solution with parasites from the parasite-free exponential solution is always supercritical, while for $\xi<1$ the bifurcation may be (the exact condition is given in that paper) subcritical. In the latter case, positive exponential solutions would exist also below the threshold and would not be always unique, in contrast to the results of this paper about equilibria. She also found that a similar pattern (but with a different condition) holds for a two-dimensional approximation of that model. The result was also confirmed for a three-dimensional (with variable aggregation) approximation [22]. Moreover, it was found that it held also for equilibria when density-dependence is assumed in the same two-dimensional approximation [21]. Therefore we were surprised of the result of Section 3 that, independently of the value of $\xi$, a positive equilibrium exists only if $R_{0}>1$ and is always unique; we do not understand fully the reasons for the difference between this result and the previous ones.

\section{REFERENCES}

[1] Anderson, R. M., and May, R. M. Regulation and stability of host-parasite populations interactions 1-2. J. Animal Ecology 47, (1978), 219-247, 249-267.

[2] Bailey, N. T. J. The elements of stochastic processes with applications to the natural sciences. Reprint of the 1964 original. (Wiley, New York, 1990).

[3] Butzer, P. L., and Berens, H. Semigroups of operators and approximation. Grund. d. math. Wiss., 145, (Springer-Verlag, Berlin-Heidelberg-New York, 1967).

[4] Desch, W., and Schappacher, W. On relatively bounded perturbations of linear $C_{0^{-}}$ semigroups. Ann. Scuola Norm. Sup. Pisa 11, (1984) 327-341

[5] Desch, W., and Schappacher, W. Linearized stability for nonlinear semigroups, in Differential equations in Banach spaces (Bologna, 1985), A. Favini and E. Obrecht (eds.), 61-73, Lecture Notes in Math., 1223 (Springer, Berlin-New York, 1986). 
[6] Desch, W., and Schappacher, W. Some generation results for perturbed semigroups, in Semigroup theory and applications (Trieste, 1987), P. Clment, S. Invernizzi, E. Mitidieri and I. I. Vrabie (eds.); 125-152, Lecture Notes in Pure and Appl. Math., 116 (Dekker, New York, 1989).

[7] Desch, W., Schappacher, W., and Zhang, K.P. Semilinear evolution equations. Houston J. Math. 15, (1989) 527-552.

[8] Diekmann, O., Heesterbeek, J. A. P., and Metz, J. A. J. On the definition and the computation of the basic reproduction ratio $R_{0}$ in models for infectious diseases in heterogeneous populations. J. Math. Biol., 28, (1990) 365-382.

[9] Diekmann, O., and Kretzschmar, M. Patterns in the effects of infectious diseases on population growth. J. Math. Biol., 29, (1991) 539-570.

[10] Engel, K., and Nagel, R. One-parameter semigroups for linear evolution equations. With contributions by S. Brendle, M. Campiti, T. Hahn, G. Metafune, G. Nickel, D. Pallara, C. Perazzoli, A. Rhandi, S. Romanelli and R. Schnaubelt. Graduate Texts in Mathematics, 194 (Springer-Verlag, New York, 2000).

[11] Hadeler, K. P., and Dietz, K. Nonlinear hyperbolic partial differential equations for the dynamics of parasite populations. Hyperbolic partial differential equations. Comput. Math. Appl. 9, (1983), no. 3, 415-430.

[12] Hadeler, K. P., and Dietz, K. Population dynamics of killing parasites which reproduce in the host, J. Math. Biol. 21, (1984) 45-65.

[13] Hudson, P.J., Rizzoli, A., Grenfell, B. T., Heesterbeek, H. and Dobson, A.P. (eds.), Ecology of Wildlife Diseases (Oxford University Press, Oxford, 2002)

[14] Iannelli, M. Mathematical theory of age-structured population dynamics. Applied Math. Monographs del CNR, n.7. (Giardini Ed., Pisa, 1995).

[15] Isham, V. Stochastic models of host-macroparasite interaction. Ann. Appl. Probab. 5, (1995) $720-740$.

[16] Kostizin, V. A. Symbiose, parasitisme et évolution (étude mathématique), Hermann, Paris, (1934). Translated in The Golden Age of Theoretical Ecology, F. Scudo and J. Ziegler(eds.), pp. 369-408, Lecture Notes in Biomathematics, Vol. 22, Springer-Verlag, Berlin, 1978.

[17] Kretzschmar, M. A renewal equation with a birth-death process as a model for parasitic infections. J. Math. Biol. 27, (1989) 191-221.

[18] Kretzschmar, M. Comparison of an infinite dimensional model for parasitic diseases with a related 2-dimensional system. J. Math. Anal. Appl. 176, (1993) 235-260.

[19] Kretschmar, M., and Adler, F. R. Aggregated distributions in models for patchy populations. Theoret. Pop. Biol. 43, (1993) 1-30.

[20] Pugliese, A. Coexistence of macroparasites without direct interactions. Theor. Pop. Biol. 57, (2000) 145-165.

[21] Pugliese, A., and Rosà, R. A 2-dimensional model for macroparasitic infections in a host with logistic growth, J. Biol. Syst. 3, (1995) 833-849.

[22] Pugliese, A., Rosà, R., and Damaggio, M. L. Analysis of a model for macroparasitic infection with variable aggregation and clumped infections, J. Math. Biol. 36, (1998) 419-447.

[23] Pugliese, A., and Tonetto, L. Well-posedness of an infinite system of partial differential equations modelling parasitic infection in an age-structured host. (2002), submitted.

[24] Roberts, M. G. and Grenfell, B. T. Mathematical models for macroparasites of wildlife, in Ecology of Infectious Diseases in Natural Populations, B.T. Grenfell and A. P. Dobson (eds.), (Cambridge Univ. Press 1995), pp. 178-208.

[25] Rosà, R., and Pugliese, A. Aggregation, stability and oscillations in different models for host-macroparasite interactions, Theor. Pop. Biol. 61, (2002) 319-334. 
[26] Webb, G. F. Theory of nonlinear age-dependent population dynamics. Pure and applied Mathematics, (Marcel Dekker, New York-Basel, 1985).

[27] Webb, G. F. A semigroup proof of the Sharpe-Lotka theorem, in Infinite-dimensional systems, F. Kappel and W. Schappacher (eds.), pp. 254-268, Lecture Notes in Mathematics, (Vol. 1076 Springer-Verlag, Berlin, 1984).

Dipartimento di Matematica - Università di Trento - Via Sommarive 14 - 38050 Povo (TN), ITALY

E-mail address: pugliese@science.unitn.it

Dipartimento di Matematica Pura e Applicata, Università di Padova, Via BelZONI 7, 35131 PADOVA, ITALY

E-mail address: lorenza@math.unipd.it 